\title{
AN EXCEPTIONAL MODEL FOR GRAND UNIFICATION
}

R. Barbieri and D.V. Nanopoulos

CERN -- Geneva

\section{ABSTRACT}

A unified model of strong and electro-weak interactions based on the exceptional group $\mathrm{E}_{6}$ is presented, with three generations of fermions and all the symmetry breaking obtained from the fermion-fermion operator. A small mass for the neutrinos is obtained in a way which may be natural. The asymptotic SU(5) relations $\mathrm{m}_{\mathrm{b}}=\mathrm{m}_{\tau}, \mathrm{m}_{\mathrm{s}}=\mathrm{m}_{\mu}$, $\mathrm{m}_{\mathrm{d}}=\mathrm{m}_{\mathrm{e}}$, as well as a prediction for the top quark mass $\mathrm{m}_{t}=\left(\mathrm{m}_{\mathrm{T}} / \mathrm{m}_{\mathrm{t}}\right) \mathrm{m}_{c} \simeq$ $\simeq 20 \mathrm{GeV}$ at the toponium mass, arise naturally from a limiting situation of no mixing angles between different generations. The smallness of the mixing angles, which may be generated by radiative corrections, implies modifications to these relations which are larger for the lighter generations. These results are the reflection on the mass matrix of the conventional fermion sector of the superheavy fermions present in the theory, e.g. through a contribution of order $\delta m \simeq(\alpha / \pi)\left(M_{W} / M_{X}\right) m_{f}$, with $m_{f}$ a superheavy fermion mass. 
1.- If colour and flavour have to live together within a simple gauge group $G$ in a truly unified theory of strong and electro-weak interactions, it is quite clear by now that we will have to live with a superheavy mass scale $M$, nearer to the Planck mass than to the masses' accessible in laboratory experiments. The arguments are easily enumerated. In order of importance they are: i) the proton quasi stability; $i i)$ the large renormalization effects needed to bring the various couplings $\alpha, \alpha_{w}, \alpha_{s}$ from their "geometrical" values to the observed ones; iii) cosmological considerations, especially connected with the baryon/entropy ratio $n_{B} / n_{\gamma} \simeq 10^{-9 \pm 1}$. The question whether the "low energy" gauge group $\operatorname{Su}(3) \times$ $\times \mathrm{SU}(2) \times \mathrm{U}(1)$ sets in already at such superlarge energies is, of course, an open one, but we will stick in the following to this simple hypothesis: The Grand Plateau ${ }^{*}$.

We have actually learned most of these arguments in the unified model based on SU(5) which was introduced ${ }^{1)}$ by requiring a minimum enlargement of the $\mathrm{SU}(3) \times \mathrm{SU}(2) \times \mathrm{U}(1)$ group. One may wonder, however, whether $\mathrm{SU}(5)$ is the full story. In fact, assuming the existence of some physics at a superlarge $M$, one may try to formulate a model starting from $M$ and then going down to the mass scale $m$ explored in present (and future?) laboratory experiments. This is what we will do in the following by exploiting the properties of the exceptional group $\left.E_{6}^{* *}\right)$. We think, in fact, that $E_{6}$ offers the best changes of unification - in the vertical direction, i.e., allowing for the family repetition - because of the following features of the model that we are going to describe:

i) automatic absence of anomalies;

ii) all the basic fermions of one generation transforming as the fundamental irreducible (27 dimensional) representation of the group;

iii) the possibility of discussing the pattern of symmetry breaking, invo1ving at least two steps

$$
G \rightarrow S U(3) \times S U(2) \times U(1) \equiv G_{1} \rightarrow S U(3) \times U(1) \equiv G_{2}
$$

*) One can envisage at least two possible departures from this hypothesis: i) a larger group than $\mathrm{SU}(3) \times \mathrm{SU}(2) \times \mathrm{U}(1)$ broken to it at energies not too far from the breaking of $S U(3) \times S U(2) \times U(1)$ itself; ii) an unbroken group manifesting itself à la QCD at energies not yet explored (prime-colour).

$* *$ ) Unified theories based on $E_{6}$ have already been explored by a number of authors, in directions different from ours. A not too incomplete list of them is found in Ref. 2). 
in terms of the fermion-fermion operator, or more explicitly in terms only of Higgs fields which transform under the gauge group in the same way as such an operator. One wants in this way to leave open the possibility of interpreting the scalars not as fundamental degrees of freedom of the basic Lagrangian, but only as the reflection of some - as yet unknown - dynamics ${ }^{*}$.

As to the first step of the breaking, occuring at superhigh energies, it will have in general consequences not only on the vector boson spectrum, leaving massless the 12 bosons of $G_{1}$, but also on the fermionic spectrum: it seems natural that every particle that "sees" (couples to) the medium triggering the superheavy breaking down to $G_{1}$ will get a superheavy mass. One is, in fact, led to formulate $\mathrm{3}^{\text {,4) }}$ the Survival Hypothesis: "Low mass fermions are those that cannot receive $G_{1}$ invariant masses". In our explicit model, after the first breaking has taken place, we will remain with the observed 15-plet of two component lefthanded fermions, massless at this stage $\left(u_{i}, u_{i}^{c}, d_{i}, d_{i}^{c}, e, e^{c}, v\right)$. Finally, the second stage of the symmetry breaking from $G_{1}$ to $G_{2}$ should reproduce the main features of the "light" bosonic and fermionic spectrum, which are respectively the following: $i)$ the relation between the $W^{ \pm}$and $Z^{0}$ masses ( $I=\frac{1}{2}$ rule) as deduced from the observed strength of neutral current weak interactions;

ii) the masslessness (or nearly so) of the left-handed neutrinos.

In fact, $E_{6}$ is selected as the unique simple group upon which a model possessing the above properties can be constructed. More precisely, previous investigations ${ }^{5)}$ restrict the simple groups which admit complex representations and are automatically anomaly free to $E_{6}$ and $s 0(4 n+2), n \geq 2$. The fermionic representation of the unifying group has to be complex, since otherwise it would be in particular real with respect to the subgroup $\mathrm{SU}(3) \times \mathrm{SU}(2) \times \mathrm{U}(1)$ and a11 femions would receive a superheavy mass, according to the Survival Hypothesis. This same hypothesis also excludes all so( $4 n+2)$ groups with $n \geq 3$, at least with fermions in the spinorial representation, which is complex and anomaly free ${ }^{* *}$ ). Such a $2^{2 n}$ dimensional representation is, in fact, real under the subgroup s0(10), - and a fortiori under $\mathrm{SU}(3) \times \mathrm{SU}(2) \times \mathrm{U}(1)-$, since the following reduction under s0(10) holds

$$
2^{2 n}=2^{2 n-5} \times 16+2^{2 n-5} \times \overline{16}
$$

*) The possibility offered by $E_{6}$ of having all the breaking needed occuring in the fermion-fermion operator has been also noticed by Ramond [see Ref. 2)].

${ }^{* *}$ ) But see footnote on $\mathrm{p} .1$, especially in connection with the prime-colour possibility. 
At this point we are left with $E_{6}$ and $S 0(10)$, and it is only in $E_{6}$, but not in $\mathrm{SO}(10)$, that the symmetry breaking needed can occur in the fermion-fermion operator (see below).

2.- $\mathrm{E}_{6}$ is the exceptional group of rank 6 with 78 generators, most of which have to be broken at superlarge mass, leaving unbroken (or nearly so) the 12 generators of $G_{1}$. The fermions are assigned to the 27 fundamental representation, so the relevant Higgs must couple to the product

$$
\underline{27} \times \underline{27}=(\overline{27}+351)_{S}+\underline{351}_{A}^{\prime} \text {. }
$$

Also to make contact with previous work, it will be useful to consider the various reductions under the subgroups $\mathrm{E}_{6} \supset \mathrm{SO}(10) \times \mathrm{U}(1) \supset \mathrm{SU}(5) \times \mathrm{U}(1) \times \mathrm{U}(1)$ which can be found in Table 1 , and are mostly obtained from

$$
\begin{aligned}
& \underline{27}=\underline{16}+\underline{10}+\underline{1} \quad[\text { under } \mathrm{s} 0(10)] \\
& \underline{16}=\underline{10}+\underline{\overline{5}}+1 \quad \quad \text { [under } \mathrm{su}(5)] \\
& \underline{10}=\underline{5}+\overline{5}
\end{aligned}
$$

In order to be able to break the group down to $G_{1}$, we need to find vectors which are stabilized by $G_{1}$ in the various Higgs representations of Eq. (1). More than that, in ordex to implement the Survival Hypothesis we need to know all the vectors contained in $\mathrm{Eq}$. (l) which are left invariant by $G_{1}$. Each of them is capable of giving a superheavy mass to some fermions by getting a superlarge vacuum expectation value (vev). All of this is most easily done by considering the embedding of $G_{1}$ in $S U(5)$, knowing that only the real representations of SU(5) contain a vector which is left invariant by $G_{1}$ : in our case only the $\underline{1}$ and the 24 of SU(5) are relevant, with the 24 breaking SU(5) down to $G_{1}$. From Table 1 , one sees that both the larger Higgs representations contained in Eq. (1) (351 and/or 351'), can undergo the superlarge breaking with the $\underline{16}$ of $\mathrm{SO}(10)$ breaking $\mathrm{E}_{6}$ down to $\mathrm{SU}(5)$, and the $\underline{24}$ of $\mathrm{SU}(5)$ breaking SU(5) down to $G_{1}$; the Higgs in the $\underline{27}$ on the contrary could not possibly do all of the superlarge breaking ${ }^{*}$. Most important for us now are

*) Actually from Table 1 one also sees that the SO(10) product $16 \times 16=10+$ $126+120$ contains only one component which is $G_{1}$ invariant. The breaking to $G_{1}$ cannot then be achieved because this same component is an $\mathrm{SU}(5)$ singlet. 
the Yukawa couplings of all the $G_{1}$ invariant Higgs components, which are easily written down by making use of Table $l^{*}$ ) in terms of the following self-explanatory notation for the 27 multiplet $\psi$ of left-handed fermions;

i) the 16 under $\mathrm{sO}(10)$

$$
\left(\nu, u_{i}, e, d_{i}, d_{i}^{c}, e^{c}, u_{i}^{c}, \nu^{c}\right) \quad(i=R, B, W)
$$

ii) the 10 under s0(10)

$$
\left(D_{i}, N, E, E^{c}, N^{c}, D_{i}^{c}\right)
$$

iii) the 1 under $S 0(10)$ denoted by $L$.

The relevant couplings are

$$
\begin{aligned}
\psi \varphi_{351} \psi & \simeq \varphi(1) L L+\varphi(16) \nu^{c} L+\varphi(126) \nu^{c} \nu^{c} \\
& +\varphi(54,24)\left(D^{c} D-\frac{3}{2} E^{c} E-\frac{3}{2} N^{c} N\right)+ \\
& +\varphi(144,24)\left(d^{c} D-\frac{3}{2} E^{c} e-\frac{3}{2} N^{c} \nu\right) \\
\psi \chi_{351^{\prime}} \psi & \simeq \chi(16) \nu^{c} L+\chi(\overrightarrow{16})\left(d^{c} D+E^{c} e+N^{c} \nu\right) \\
& +\chi(45)\left(D^{c} D+E^{c} E+N^{c} N\right)+ \\
& +\chi(45,24)\left(D^{c} D-\frac{3}{2} E^{c} E-\frac{3}{2} N^{c} N\right) \\
& +\chi(144,24)\left(d^{c} D-\frac{3}{2} E^{c} e-\frac{3}{2} N^{c} \nu\right)
\end{aligned}
$$

Here $\varphi(a)(X(a))$ denotes the component of $\varphi(X)$ transforming as a under $S 0(10)$ and 1 under $\mathrm{SU}(5)$, whereas $\varphi(a, 24)(\chi(a, 24))$ denotes an a under so(10) and a 24 component under $S U(5)$. Once all these components have got a superlarge vev, it is straightforward to see that one is left with the relevant 15-plet of massless particles, whereas a11 the other fermions get a superheavy mass. Basically what happens is that the $\underline{27}$ of $\mathrm{E}_{6}$ is reduced as $\underline{27}=\underline{10}+$ $\overline{5}+\overline{5}+\underline{5}+\underline{1}+\underline{1}$ under $\mathrm{SU}(5)$ and the self-conjugate part of this reducible representation $(\underline{5}+\underline{\overline{5}}+\underline{1}+\underline{1})$ gets a superheavy mass.

*) When the reductions under $S O(10)$ and $S U(5)$ are not sufficient to get all the Clebsch-Gordan coefficients, the reduction under the subgroup $\mathrm{SU}(3) \otimes$ $\otimes \mathrm{SU}(3) \otimes \mathrm{SU}(3)$ is relevant, with $\underline{27}=(3,3,1)+(\overline{3}, 1, \overline{3})+(1, \overline{3}, 3)$. 
In order to discuss the effect of the further breaking on the surviving 15 massless fields, it is useful to describe them in terms of the original fields as follows:

i) the $Q=2 / 3$ quark is left untouched $\left(u, u^{c}\right)$;

ii) including also the generation degree of freedom, from Eqs (1)-(3) the $Q=-1 / 3$ mass matrix can be written as

$$
\left(d^{c} D^{c}\right)\left(\begin{array}{ll}
0 & A \\
0 & B
\end{array}\right)\left(\begin{array}{l}
d \\
D
\end{array}\right)
$$

where each field represents an $N$ component vector in generation space and the entries of the mass matrix are themselves $N \times N$ matrices. The diagonalization can be performed by the bi-unitary transformation

$$
\left(\begin{array}{l}
d \\
D
\end{array}\right)=\left(\begin{array}{cc}
11 & 0 \\
0 & v
\end{array}\right)\left(\begin{array}{l}
d_{0} \\
D_{0}
\end{array}\right) ; \quad\left(d^{c} D^{c}\right)=\left(\begin{array}{ll}
d_{0}^{c} & D_{0}^{c}
\end{array}\right)\left(\begin{array}{cc}
U_{11}^{c} & U_{12}^{c} \\
U_{21}^{c} & U_{22}^{c}
\end{array}\right)
$$

with the massless eigenvectors being described by

$$
\left(d_{0}, d_{0}^{c}\right) \equiv\left(d, d^{c} U_{11}^{c^{+}}+D^{c} U_{12}^{c^{+}}\right)
$$

and the orthogonal combinations being superheavy;

iii) Similarly the $Q=-1$ lepton mass matrix is diagonalized by

$$
\left(\begin{array}{l}
e \\
E
\end{array}\right)=\left(\begin{array}{ll}
V_{11} & V_{12} \\
V_{21} & V_{22}
\end{array}\right)\left(\begin{array}{l}
e_{0} \\
E_{0}
\end{array}\right),\left(e^{c} E^{c}\right)=\left(e_{0}^{c} E_{0}^{c}\right)\left(\begin{array}{ll}
\mathbb{1} & 0 \\
0 & V^{c}
\end{array}\right)
$$

so that the massless combinations are

$$
\left(e_{0}, e_{0}^{c}\right) \equiv\left(V_{11}^{+} e+V_{21}^{+} E, e^{c}\right)
$$


iv) In the neutral lepton sector one gets, per generation, four superheavy two-component particles and one massless two-component neutrino $\nu_{0}=$ $=\mathrm{V}_{11}^{+} \nu+\mathrm{V}_{21}^{+} \mathrm{N}$. The matrices $\mathrm{V}_{11}, \mathrm{~V}_{21}$ are the same as those entering in Eq. (8). More precisely the $5 \times 5$ neutral lepton mass matrix in split into the $2 \times 2$ sector $\left(\nu_{c}, \mathrm{~L}\right)$ which gives two superheavy Majorana particles and the $3 \times 3$ sector $\left(\nu, N, N^{c}\right)$ out of which comes the above massless neutrino plus a superheavy Dirac particle.

Notice that the SU(2) weak currents coincide at this level with the conventional ones in the zero mass sector. Notice also that if only one $\varphi_{351}$ symmetric representation is present and no $\chi_{351}$, antisymmetric representation which would be enough to give superheavy masses to all unobserved fermions even with three generations - one would have

$$
\begin{array}{ll}
U_{11}^{c}=\cos \theta u & V_{11}=\cos \theta u^{\top} \\
U_{12}^{c}=\operatorname{sen} \theta U & V_{21}=\operatorname{sen} \theta U^{\top}
\end{array}
$$

with $U$ a unitary matrix in generation space. This relation between the rotation matrices of the $Q=-1 / 3$ and $Q=-1$ sectors is a consequence of the Clebsch-Gordan coefficients of $\varphi_{351}$ in Eq. (2), which are different from those of $X_{351}$, , Eq. (3). These properties of $\varphi_{351}$ will be used in the following.

3.- We go now to the last stage of symmetry breaking from $G_{2}$ to $G_{2}$, which we envisage as follows. The smallest $\underline{27}$ Biggs representation contained in Eq. (1), which could not possibly do all of the first stage of breaking since it does not contain a vector stabilized by $G_{1}$, is first uncoupled from the higher representations, and it undergoes the breaking of the group down to $G_{2}$ with all its $G_{2}$ invariant components getting vey's of ordex $v_{0} \simeq \mathrm{m} \simeq 100 \mathrm{Gev}^{*}$.

*) In the absence of coupling between the higher and the lower dimensional representations this is not only possible but actually plausible, according to an idea put forward by E11is, Gaillard, Peterman and Sachrajda6). With no mass term and all dimensionless couplings of the same order, say $\mathrm{g}^{2}$, in the Higgs Lagrangian relevant at some point near the Planck mass, the hierarchy $M \gg m$ may then come from radiative corrections with the Higgs representations with larger non-Abelian charges developing vev's of order $v \simeq M$ much greater than those of the representation with lower dimensionality, due to the different behaviour under the renormalization group of their respective quartic couplings. 
A situation like this one (all the vev's of the $G_{1}$ invariant components of $\varphi_{351}$, $X_{351}$, - and no others - different from zero and $\simeq v$; all the vev's of the $G_{2}$ invariant components of $\mathrm{H}_{27} \simeq \mathrm{v}_{0}<<\mathrm{v}$ ) corresponds precisely to the requirements that we formulated at the beginning for the second stage of symmetry breaking: the masslessness (or nearly so) of the left-handed neutrinos and the $I=\frac{1}{2}$ rule in neutral current weak interactions is expressed, in terms of weak. SU(2), by the fact that the 27 Higgs representation contains only Higgs doublets (or singlets), whereas the SU(2) triplets contained in the $\underline{351}$ and $351^{\prime}$ do not get vev's (or negligibly sma1l ones).

Actually a coupling between $\mathrm{H}_{27}$ and $\varphi_{351}, \chi_{351}$, must be present for a number of good reasons (radiative corrections, unwanted light Higgses, etc.). As is well known, such a coupling has to be tuned very carefully in order not to destroy the hierarchy itself. Most importantly for us this coupling will also cause non-zexo vev's $\left\langle\varphi_{351}\right\rangle$ and $\left\langle\chi_{351},\right\rangle$, which break $G_{1}$ to $G_{2}$, with some components (SU(2) triplets) getting in general unwanted vev's. We argue, however, in the following that such components will be negligibly small. When a coupling interaction $\lambda V(\varphi, \chi, H)$ is added to the uncoupled potentials $V(H), V(\varphi, \chi)$, respecting the discrete symmetry $\mathrm{H} \rightarrow-\mathrm{H}$, there will be an extremum of the combined potentials which connects up with the previous solution in the limit as $\lambda \rightarrow 0$; for sufficiently small $\lambda$ this will still be the absolute minimum, and hence that which is chosen in the "real world". As we said, the $G_{2}$ singlet, $G_{1}$ non-singlet components of $\varphi$ and $X$ - call them $\varepsilon$-will also be different from zero. The leading dependence on $\varepsilon$ of the potentials will in general be of the form

$$
V(\varphi, x) \simeq v^{2} \epsilon^{2} \quad ; V(\varphi, x, H) \simeq v_{0}^{2} \epsilon v
$$

because of $G_{1}$ invariance and $H \rightarrow-H$ symetry, respectively. Therefore, the stationarity condition on $\varepsilon$ for the combined potentials leads to

$$
\epsilon \simeq \frac{v_{0}^{2}}{v}
$$

which in turns means that the breaking of $G_{1}$ down to $G_{2}$ by the Higgs fields in the larger representations is much less then that due to the 27 representation of Eq. (1). This argument, which of course will have to be substantiated by an explicit study of the possible invariant Higgs interactions, does not seem 
to depend on any special choice of the potentials and, as such, is likely to persist "naturally" also when radiative corrections are taken into account"). More precisely, radiative corrections may break the $\mathrm{H} \rightarrow-\mathrm{H}$ invariance of the Higgs potentials; in such a case the SU(2) doublet $\varepsilon$ components may get vev's of order $v_{0}$ since $V(\varphi, \chi, H)$ will contain induced terms behaving like. $\varepsilon \mathrm{v}_{0} \mathrm{v}^{2}$, but this will still not be the case for the triplet $\varepsilon$ components because of $\mathrm{SU}(2)$ invariance ${ }^{* *}$ ).

4.- To discuss "light" fermion masses explicitly, in particular the neutrino, we write down the Yukawa couplings of a11 $G_{2}$ singlet components in the $\underline{27}$ of Higgs, which all acquire, by assumption, a non-vanishing vev $\simeq \mathrm{v}_{0}$. They are

$$
\begin{aligned}
\psi H_{27} \psi & \simeq H(1,1)\left(D^{c} D+E^{c} E+N^{c} N\right)+H(16,1)\left(d^{c} D+E^{c} e+N_{\gamma}^{c}\right) \\
& +H(16, \overline{5})\left(D^{c} d+e^{c} E+N^{c} \nu^{c}\right)+H(10, \overline{5})\left(d^{c} d+e^{c} e+N^{c} c\right) \\
& +H(10,5)\left(u^{c} u+\gamma^{c} \nu+L N\right)
\end{aligned}
$$

where, as above, $\mathrm{H}(\mathrm{a}, \mathrm{b})$ denotes a component of $\mathrm{H}_{27}$ which transforms as $\underline{\text { a }}$ under $\mathrm{SO}(10)$ and $\underline{b}$ under $\mathrm{SU}(5)$. The $\mathrm{SU}(5)$ singlets modify, in a completely negligible way, the masses of superheavy fermions discussed above, whereas the $\underline{5}$ and $\overline{5}$ components under $\mathrm{SU}(5)$ give masses to all the $15-\mathrm{plet}$ of light fermions as follows. First in the $5 \times 5$ mass matrix of the neutral leptons the SU(5) non-singlets in $\mathrm{Eq}$. (12) couple the $\left(\nu_{c}, \mathrm{~L}\right)$ superheavy sector with the $\left(\nu, N, N^{C}\right)$ sector containing the massless neutrino; however, since the separate sectors axe left totally unchanged, the new diagonalization leads to a Majorana neutrino of mass $\mathrm{m}^{2} / \mathrm{M}$ with a negligibly small mixing $(\simeq \mathrm{m} / \mathrm{M})$ with. right-handed components. True enough, the $\left(\nu, N, N^{c}\right)$ - sector itself is modified by the $G_{1}$ non-singlet vev's (SU(2) triplets) in the higher Higgs representations, but the situation concerning the light neutxino mass does not change

*) An explicit example of the mechanism discussed here in general is analysed in Ref. 7).

**) The difficulty pointed out in Ref. 4) should be overcome in this way: all undesirable infinities are absorbable in the Higgs potential parameters. 
since these vev's are themselves of order $\varepsilon \simeq \mathrm{v}_{0}^{2} / \mathrm{v} \simeq \mathrm{m}^{2} / \mathrm{M}$. Taking for $\mathrm{m} a$ rms quark or lepton mass $\mathrm{m} \simeq 2 \mathrm{GeV}$, and $M \simeq 10^{15} \mathrm{GeV}$, one is led to an estimate for the Majorana neutrino mass $m_{v} \simeq 10^{-5} \mathrm{eV}$, which may have striking phenomenological implications in solar neutrino physics.

As to the $Q=-1 / 3$ quark and the $Q=-1$ lepton masses, Eq. (12) possesses the global SU(4) symmetry which has been used in SU(5) to get the mass relations $\mathrm{m}_{\mathrm{b}}=\mathrm{m}_{\tau}, \mathrm{m}_{\mathrm{s}}=\mathrm{m}_{\mu}, \mathrm{m}_{\mathrm{d}}=\mathrm{m}_{\mathrm{e}}$ at superheavy energies. Such a symetry is, however, no longer relevant in general for the zero mass eigenstates $\left(d_{0}, d_{0}^{c}\right),\left(e_{0}, e_{0}^{c}\right)$ in the $G_{1}$ symmetric limit discussed above, since $U_{11}^{c} \neq V_{11}, U_{21}^{c} \neq V_{12}$ in Eqs (6) and (8). Rewriting the relevant components of Eq. (12) in terms of such eigenstates one easily gets, if only one $\mathrm{H}_{27}$ is present, the following mass matrices in generation space for the $Q=-1 / 3$ quarks and the $Q=-1$ leptons

$$
\begin{aligned}
& M^{(-1 / 3)}=\left(a U_{11}^{c}+b U_{12}^{c}\right) M^{(2 / 3)} \\
& M^{(-1)}=M^{(2 / 3)}\left(a V_{11}+b V_{21}\right)
\end{aligned}
$$

where $M^{(2 / 3)}$ is the $Q=2 / 3$ quark mass matrix, and $a, b$ are complex numbers related to the vev's of the Higgs field in Eq. (12). We are neglecting here small mixings with the superheavy sector. Some interesting consequences can be drawn from these last equations. Even with only one Higgs representation $\left(\mathrm{H}_{27}\right)$ coupled to the "light" fermion sector, one has a non-trivial Kobayashi-Maskawa (KM) matrix connecting the different generations, since $\left(\mathrm{aU}_{11}^{\mathrm{c}}+\mathrm{bU}_{12}\right)$ is not in general proportional to a unitary matrix ${ }^{*}$ and therefore the relative diagonalization of $\mathrm{M}^{(-1 / 3)}$ versus $\mathrm{M}^{(2 / 3)}$ cannot be simply achieved by a unitary transformation on the right-handed fields $\mathrm{d}^{\mathrm{C}}$. The mixing angles in the superheavy sector are reflected in the light sector. Actually a unit KM matrix is obtained if only the $\varphi_{351}$ - symmetric representation gets superlarge vey's, since in this case

$$
M^{(-1 / 3)}=(a \cos \theta+b \operatorname{sen} \theta) u M^{(2 / 3)}
$$

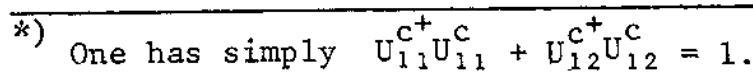


in view of Eq. (9), with $\mathcal{U}^{+} \dot{u}=1$. In such a case, furthermore, the eigenvalues of $M^{(-1)}$ are equal to those of $M^{(-1 / 3)}$, since

$$
M^{(-1)}=(a \cos \theta+b \operatorname{sen} \theta) M^{(2 / 3)} u^{\top}
$$

One is then led to speculate that a perturbative deviation from this situation would introduce the relatively small $\mathrm{KM}$ angles and, at the same time, would correct the relations $\mathrm{m}_{\mathrm{b}}=\mathrm{m}_{\tau}, \mathrm{m}_{\mathrm{s}}=\mathrm{m}_{\mu}, \mathrm{m}_{\mathrm{d}}=\mathrm{m}_{\mathrm{e}}$ by an increasing amount for the lighter generations, as is phenomenologically required. Such a perturbation is, in fact, present due to radiative corrections induced on the various mass matrices by the exchange of superheavy bosons connecting the "light" with the superheavy fermion sector $^{*}$ ), (Fig. 1). Their relative currents do have, in fact, non-vanishing zeroth order angles. The contribution from the general diagram of Fig. 1, which is finite because of the mixing needed between gauge bosons with different weak SU(2) properties, can be estimated as

$$
\delta m=\frac{\alpha}{\pi} \frac{m_{b}}{M} M_{w}
$$

where $m_{f}$ is a superheavy fermion mass, $M$ is a superheavy boson mass and $M_{W}$ is the conventional SU(2) gauge boson mass ${ }^{* *}$ ). Also relatively small vev's of the $x_{351} \mu^{-}$antisymmetric representation could trigger this perturbation, but the possibility of doing all of this by radiative corrections is, of course, much more appealing.

This possible correlation seems interesting to us. Through the reflection of the superheavy fermion sector on the mass matrix of the conventional fermions the following situation emerges: i) $\mathrm{m}_{b}=\mathrm{m}_{\tau}, \mathrm{m}_{\mathrm{s}}=\mathrm{m}_{\mu}, \mathrm{m}_{\mathrm{d}}=\mathrm{m}_{\mathrm{e}}$ are in general only approximate relations; $i i)$ the non-vanishing but small generalized Cabibbo angles indicate that these relations get corrections which are larger for the lighter generations; $\mathrm{iii)}$ as a matter of fact, for the heaviest generation, well separated in mass from the others, this correction is negligible, which fits nicely with the phenomenological success of $m_{b}=m_{\tau}$ and the three-generation picture. It is worth recalling here that precisely three families (or four at most) are needed to renormalize $\mathrm{m}_{\mathrm{b}}=\mathrm{m}_{\tau}$ from superheavy energies to the observed value $m_{b} \simeq 2.5 m_{\tau}$ at the $r$ mass 7 .

*) Notice that without a superheavy fermion sector, the absence of mixing angles $* *)$ in zeroth order implies a generation gap forever.

We believe that Eq. (16) is a general feature of theories containing superheavy fermions. Left and Right components of superheavy fermions transform in the same way under weak SU(2) in contrast to "light" fermions, as demanded by the Survival Hypothesis. A detailed analysis will be given elsewhere. 
Finally we notice that Eqs (14) and (15) also imply the following relation:

$$
m_{t}: m_{r}=m_{c}: m_{\mu}=m_{u}: m_{e}
$$

which, according to the previous picture, will also receive corrections more important for the lighter generations. To the extent that $m_{b}=m_{\tau}$ and $m_{s}=m_{\mu}$ at superhigh energies agree with phenomenological information one is led to the prediction

$$
m_{t}=\frac{m_{\tau}}{m_{\mu}} m_{c}
$$

At the toponium mass, i.e., taking into account finite renormalization effects when extrapolating from the charmonium region, this relation implies $\mathrm{m}_{t} \simeq 20 \mathrm{GeV}$ for a mass of the charmed quark $m_{c} \simeq 1.5 \mathrm{GeV}$.

5.- In conclusion we would like to summarize the reasons why we think that $\mathrm{E}_{6}$ offers, along the lines that we have discussed, the best prospects for grand unification. First, there is the fermion assignment to the lowest irreducible representation of the group which allows one to achieve maximum symetry between quarks and leptons. In fact, $\mathrm{E}_{6}$ has in it the Pati-Salam ${ }^{8)} \mathrm{SU}(4)$ extension of the colour group, which really puts quarks and leptons on the same footing; such a subgroup is also contained in $\mathrm{SO}(10)$, but in this case one has to use the spinorial 16 representation of the group instead of the fundamental one. In view of the reduction of the $\underline{27}$ of $\mathrm{E}_{6}$ under $\mathrm{SO}(10)$, it becomes clear why this has to be the case. Furthermore, the implementation of the Survival Hypothesis in $E_{6}$ allows us to understand, in a natural way, why left-and right-handed components of the observed fermions belong to different representations of the weak $\mathrm{SU}(2)$ group (doublets and singlets respectively), such that it is impossible to create $G_{1}$ invariant mass terms out of these fermions; in fact, for the same reason this pattern has to repeat itself in each generation, barring the introduction of ugly higher representations.

We then have superheavy fermions which go together with superheavy bosons and superheavy Higgsons to make a world of particles quite symetrical with respect to the "low energy" ones. In fact, we find it interesting that the mixing angles in the superheavy fermion sector are reflected in the conventional sector. This in turns allows us to discuss a realistic model for masses and angles 
with only one Higgs representation $\left(\mathrm{H}_{27}\right)$ coupled to the low energy fermions, which is, in many respects, the most natural situation $\left.{ }^{*}\right)$. The generation of mixing angles by radiative corrections in the $\mathrm{SU}(2)$ weak currents is also a distinguished feature of the superheavy fermion reflection on the "light" ones. Do we finally understand the small deviations from unity of the KM matrix?

Finally the possibility of having all breaking done through the fermionfermion operator appears interesting. This makes it more plausible that the breaking occurs in a dynamical way and gives us some insight on how the very different vev's distribute themselves between the various Higgs components. In this way we have argued how the "second hierarchy" of the neutrino masses $m_{\mathcal{V}} / \mathrm{m} \simeq \mathrm{m} / \mathrm{M}$, (and the $I=\frac{1}{2}$ rule in neutral current weak interaction), could possibly be understood without simply postulating the absence of some Higgs fields. Three very different mass scales appear in the theory $v \gg v_{0} \gg \varepsilon$, the last one possibly being understood in terms of the others, $\varepsilon \simeq v_{0}^{2} / v$. Actually, we were led to the model described in this paper by addressing our attention to the neutrino mass problem ${ }^{4)}$. Since we are believers in the Gauge Symmetry Dogma: "Exact symmetries in nature are always local", we consider by now a further merit of unified theories the possibility of discussing massive neutrinos. After baryon conservation and lepton conservation, it is (B-L) conservation which breaks down by an amount which is - to some extent - calculable.

Of more phenomenological interest are questions like the approximate relation between $Q=-1 / 3$ and $Q=-1$ fermion masses, the prediction for the top quark mass and the possible connection with the KM matrix. A11 of these questions, together with some others that we have not treated explicitly, - e.g., the possibility of obtaining $\sin ^{2} \theta_{W}=0.23$ by modifying the gauge hierarchy structure in $E_{6}$ at superhigh energy 10$)$ - could in the future favour an enlargement of $S U(5)$ to $E_{6}$ not primarily on an aesthetic basis, but also from a strict phenomenological point of view, as is perhaps already indicated.

\section{ACKNOWLEDGEMENTS}

It is a pleasure to thank for discussions F. Buccella, G. Girardi, M. Peskin, J. Prentki, C. Savoy, P. Sorba, H. Ruegg and J. Ellis, who also read the manuscript.

*) See Ref. 9) for a list of the problems in using more than one Higgs representation to get fermion masses in grand unified theories. 
Table 1

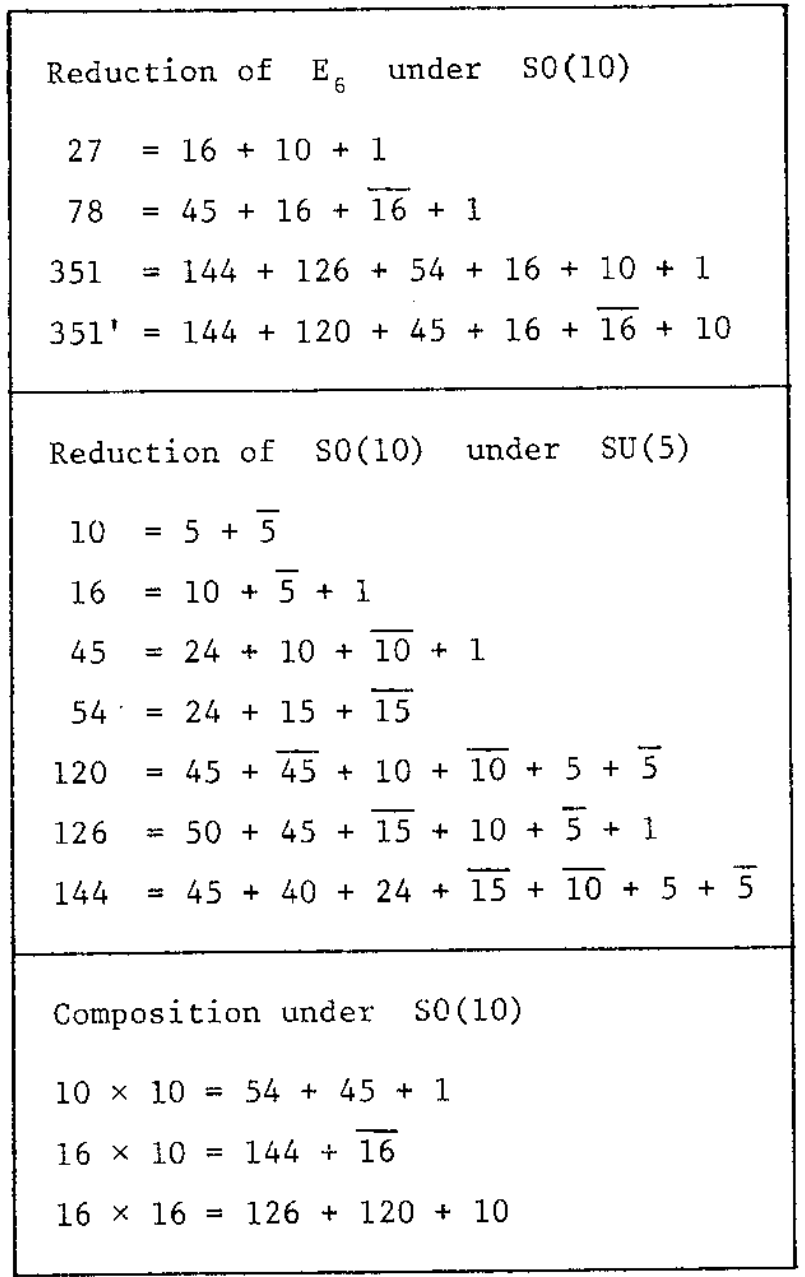




\section{REFERENCES}

1) H. Georgi and S.L. Glashow, Phys. Rev. Letters 32 (1974) 438.

2) F. Gürsey, P. Ramond and P. Sikivie, Phys. Lett. 60B (1976) 177;

F. Gürsey and M. Serdaroglu, Nuovo Cimento Letters 21 (1978) 28;

Y. Achiman and B. Stech, Phys. Lett. 77B (1978) 389;

Q. Shafi, Phys. Lett. 79B (1978) 301;

P. Ramond, Caltech preprint CALT-68-709 (1979);

H. Ruegg and T. Schücker, Nucl. Phys. B161 (1979) 388.

3) H. Georgi, Nucl. Phys. B156 (1979) 126.

4) R. Barbieri, D.V. Nanopoulos, G. Morchio and F. Strocchi, CERN preprint TH. 2776 (1979), to appear in Phys. Lett. B.

5) H. Georgi and S.L. Glashow, Phys. Rev. D6 (1973) 429;

M. Ge11-Mann, P. Ramond and R. Slansky, Rev. Mod. Phys. 50 (1978) 721.

6) J. Ellis, M.K. Gaillard, A. Peterman and C. Sachrajda, CERN preprint TH.2696 (1979).

7) A. Buras, J. Ellis, M.K. Gaillard and D.V. Nanopoulos, Nucl. Phys. B135 (1978) 66.

8) J.C. Pati and A. Salam, Phys. Rev. D10 (1974) 275.

9) J. E1lis and M.K. Gaillatd, Phys. Lett. 88B (1979) 315.

10) H. Georgi and D.V. Nanopoulos, Nuc1. Phys. B159 (1979) 16.

\section{FIGURE CAPTION}

Fig. 1 : A contribution to the "light" fermion masses. The internal double line stands for a superheavy fexmion; the wayy Iine for a superheavy gauge boson. 


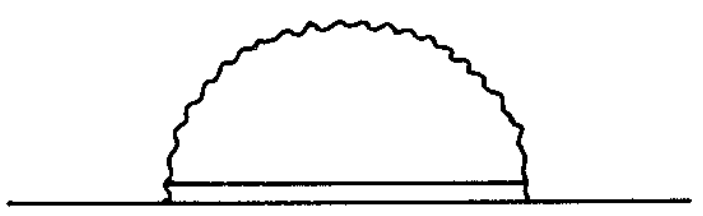

FIG.1 

18 